\title{
Arrayed MC-CDMA Reception in Space-Time Diffused Multipath Vector Channels
}

\author{
Farrukh Rashid and Athanassios Manikas \\ Communications and Signal Processing Research Group \\ Department of Electrical and Electronic Engineering \\ Imperial College London
}

\begin{abstract}
Multi-Carrier Code Division Multiple Access (MC-CDMA) is a modulation scheme that combines the advantages of OFDM and CDMA to provide robustness against frequency selectivity in wireless channels. Arrayed MC-CDMA systems combine MC-CDMA and antenna array technology to harness the spatial and temporal 'signatures' of received signals, thus making it possible to realize high transmission rates envisioned for next generation wireless communications. Localized scattering, which occurs for each multipath, motivates the frequency selective wireless channel to be modelled as a diffused vector channel. In this paper, space-time diffused vector channels for arrayed MC-CDMA systems are modelled and analyzed. Simulation studies show that the use of this diffused channel modelling in arrayed MC-CDMA receivers yields better bit error rate (BER) and $\mathrm{SNIR}_{\text {out }}$ performance than receivers that ignore the presence of spatial and/or temporal diffusion.
\end{abstract}

\section{Index Terms}

Antenna arrays, MC-CDMA, channel estimation, multipath channels, spatial diffusion, angular spread.

\section{INTRODUCTION}

Multi-Carrier Code Division Multiple Access (MC-CDMA) has been proposed for the future $4 \mathrm{G}$ wireless communication systems because it combines the advantages of OFDM and CDMA to provide robustness against frequency selectivity and channel delay spread. The use of antenna 
arrays at the receiver exploits the spatial domain to provide an extra layer of co-channel interference cancellation and new ways of handling unwanted channel effects [1]. Arrayed MC-CDMA systems combine MC-CDMA and antenna array technology to harness the spatial and temporal information of received signals in multipath channels. This synergy makes it possible to realize high transmission rates envisioned for next generation wireless communications [2].

In arrayed MC-CDMA systems, the frequency selective wireless channel is generally modelled as a Scalar-Input-Vector-Output (SIVO) channel. In the SIVO channel model, it is assumed that each multipath has distinct spatial and temporal signatures, with each multipath appearing at the receiver's antenna array as a point-like source [3]. In practical wireless channels, however, localized scattering of the multipaths invalidates the point-source assumption used by the conventional algorithms. This paper lifts the point-source assumption for arrayed MC-CDMA communication systems and considers the diffused channel scenario.

Existing literature on diffused multipath vector channels focuses mainly on DS-CDMA based wireless communication systems. In [4], diffused channel framework for DS-CDMA systems is presented and a subspace approach is proposed to estimate vector channels having diffusion in the spatial domain. Two receiver techniques for DS-CDMA systems are proposed in [5] to effectively remove the perturbation due to such multipath spatial diffusion. However, no such work has been reported in the literature for arrayed multicarrier systems operating in multipath channels that are diffused both in space and time. This paper characterizes the diffused multipath vector channel for arrayed MC-CDMA systems and proposes a subspace-based arrayed MC-CDMA receiver for the space-time diffused wireless channel. The main emphasis of the paper will be on the modelling of the space-time diffused wireless channel in order to design the receiver's weight vector more accurately.

The paper is organized as follows. First, the system model of an arrayed MC-CDMA communication system, including the transceiver structure and the point-source channel model, is described in Section II. The concept of diffused Spatial-Temporal ARray (STAR) manifold vector is then used in Section III to accurately model the diffused multipath vector channel in terms of the spatial and temporal parameters. Computer simulation studies are presented in Section IV to compare the performance of the arrayed MC-CDMA receivers that use the diffused channel model, to receivers that use the point-source channel model (and hence ignore the presence of spatial and temporal diffusion). Finally, the paper is concluded in Section V. 


\section{System ARChitecture}

Consider the uplink of an arrayed MC-CDMA wireless communication system where the mobile users transmit their signals to the base station. Each transmitter has a single transmitting antenna, but the receiver is equipped with a set of $N$ antennas. To simplify the model, it is assumed that the carrier frequency offset has been estimated and compensated. The system contains a total of $M$ transmitting users and the number of subcarriers is $N_{s c}$.

\section{A. MC-CDMA Transmitter}

The block diagram of an arrayed MC-CDMA communication system operating in a space-time diffused vector channel is shown in Fig. 1. With reference to this figure, at point A, the $i$-th user produces a sequence of unit energy Binary Phase Shift Keyed (BPSK) data symbols $\left\{\mathrm{a}_{i}[n] \in\right.$ $\{+1,-1\}, \forall n \in \mathcal{N}\}$ with symbol duration $T_{c s}$. The $i$-th user is assigned a unique PN sequence of \pm 1 's. In this paper, the PN-code period (spreading gain) is assumed to be equal to the number of subcarriers, $N_{s c}$, with one period represented by the vector $\underline{\alpha}_{i}=\left[\alpha_{i}[1], \ldots, \alpha_{i}[k] \ldots, \alpha_{i}\left[N_{s c}\right]\right]^{T}$.

The $n$-th data symbol of the $i$-th user, $\mathrm{a}_{i}[n]$, is processed by the baseband MC-CDMA transmitter as follows. The $n$-th channel symbol $\mathrm{a}_{i}[n]$ is first copied into $N_{s c}$ parallel streams. The $k$-th copy is then multiplied by the corresponding $k$-th chip of $\underline{\alpha}_{i}$, and the product then modulates the $k$-th subcarrier with frequency given by $F_{k} \triangleq k \Delta F$, where $\Delta F \triangleq 1 / T_{c s}$ is the subcarrier separation. The modulated subcarriers are then pulse-shaped and summed up to produce the baseband MC-CDMA transmitted signal $m_{i}(t)$ (see point B in Fig. 1) defined as follows

$$
\begin{aligned}
m_{i}(t)= & \sum_{k=1}^{N_{s c}} \alpha_{i}[k] \mathrm{a}_{i}[n] p(t) \exp (j 2 \pi k \Delta F t) \\
& n T_{c s} \leq t \leq(n+1) T_{c s}
\end{aligned}
$$

where $p(t)$ is a rectangular pulse of duration $T_{c s}$.

Finally after upconversion, the $i$-th user's transmitted signal (at point $\mathrm{C}$ ) is given by

$$
\text { Transmitted signal }=\sqrt{P_{i}} m_{i}(t) \exp \left(j 2 \pi F_{c} t\right)
$$

where $F_{c}$ is the carrier frequency and $P_{i}$ is the transmitted power. 


\section{B. Point-Source Channel Model}

This sub-section describes the conventional point-source channel model, where each multipath is considered as a point-source. The transmitted MC-CDMA signal is assumed to experience a wireless channel that is fading and multipath dispersive, so that it exhibits frequency selectivity. It is slowly-varying such that its frequency response is constant over the duration of $L$ MC-CDMA symbols.

The $i$-th user's transmitted signal arrives at the receiver via $K_{i}$ multipaths, each with its own fading coefficient, direction of arrival (DOA) and time of arrival (TOA). Although each multipath has a certain degree of spatial and temporal spread (and hence is diffused), the temporal and spatial diffusion is often considered to be small. The point-source channel model ignores this diffusion and treats each multipath as a point source.

Without any loss of generality, it is assumed that all transmitters are in the far-field of the receive-array, and the transmitters and receive-array are stationary and co-planar. Hence, using the plane-wave propagation, the array response vector (i.e. the array manifold vector) of the $j$-th path of the $i$-th user at the $k$-th subcarrier is given by

$$
\underline{S}_{k}\left(\theta_{i j}\right)=\exp \left(\frac{-j 2 \pi\left(F_{c}+F_{k}\right)}{c}\left[\underline{r}_{1}, \underline{r}_{2}, \ldots, \underline{r}_{N}\right]^{T} \underline{u}\left(\theta_{i j}\right)\right) \in \mathcal{C}^{N \times 1}
$$

where the vectors $\underline{r}_{1}, \underline{r}_{2}, \ldots, \underline{r}_{N}$ contain the antenna positions in Cartesian coordinates, $\exp (\underline{a})$ denotes the element-by-element exponential of the vector $\underline{a}$, $\underline{u}\left(\theta_{i j}\right) \triangleq\left[\cos \left(\theta_{i j}\right), \sin \left(\theta_{i j}\right), 0\right]^{T}$ and $c$ is the speed of light. Note that the array manifold vector is a function of the DOA $\theta_{i j}$, the subcarrier frequency $F_{k}$, and the receiver array geometry.

In this paper, the array aperture is assumed to be small enough for the narrowband assumption to hold. Narrowband assumption implies that $1 / T_{c s} \ll F_{c}$ and, for a particular path, all subcarriers have the same array manifold vector, i.e.

$$
\underline{S}\left(\theta_{i j}\right) \triangleq \underline{S}_{k}\left(\theta_{i j}\right) \forall k \in 1, \ldots, N_{s c}
$$

For an antenna array of $N$ elements used at the receiver, Fig. 2 represents the baseband pointsource Scalar-Input-Vector-Output (SIVO) channel model for the $i$-th user, where the $j$-th path has path delay $\tau_{i j}$, direction of arrival $\theta_{i j}$ and fading coefficient (up to the array reference point) $\beta_{i j}$. 
The baseband received signal-vector $\underline{x}_{i}(t)$ at the output of the SIVO channel of the $i$-th user is given by

$$
\underline{x}_{i}(t)=\sum_{j=1}^{K_{i}} \beta_{i j} \underline{S}\left(\theta_{i j}\right) m_{i}\left(t-\tau_{i j}\right) \in \mathcal{C}^{N \times 1}
$$

where $\beta_{i j}$ has absorbed the factor $\sqrt{P_{i}} \exp \left(-j 2 \pi F_{c} \tau_{i j}\right)$ and the total signal-vector due to $M$ users is given by

$$
\underline{x}(t)=\sum_{i=1}^{M} \sum_{j=1}^{K_{i}} \beta_{i j} \underline{S}\left(\theta_{i j}\right) m_{i}\left(t-\tau_{i j}\right)+\underline{\mathrm{n}}(t) \in \mathcal{C}^{N \times 1}
$$

where $\underline{\mathrm{n}}(t)$ represents isotropic complex AWGN. An equivalent expression for $\underline{x}(t)$ using the diffused SIVO channel will be given in Section III.

\section{Receiver Architecture}

At the receiver, the received signal is first down-converted and the baseband signal-vector $\underline{x}(t)$ is then sampled at a rate of $1 / T_{s}$, where $T_{s}=T_{c s} / N_{s c}$, to obtain $N_{s c}$ samples per channel symbol period per antenna. However, the delay $\tau_{i j}$ of the $j$-th path of the $i$-th user is quantized into integer and fractional component, i.e. $\tau_{i j}=\left(l_{i j}+\psi_{i j}\right) T_{s}$ with $l_{i j} \in 0,1, \ldots, N_{s c}-1$ and $\psi_{i j} \in[0,1)$, and the phase shift due to the fractional part, $\exp \left(-j 2 \pi F_{k} \psi_{i j} T_{s}\right)$, is absorbed into the complex path coefficient $\beta_{i j}$. Note that, in order to simplify the analysis, the path delay $\tau_{i j}$ has been assumed to lie within the range $\left[0, T_{c s}\right)$. The front-end of the MC-CDMA receiver for the $i$-th user is similar to that in [3] and consists of $N$ tapped delay lines (TDLs) each of length $2 N_{s c}$ in order to ensure that (due to lack of synchronization) one whole data symbol of the desired user is captured within this $2 N_{s c}$ interval.

The time variation of the $k$-th subcarrier after undergoing a path delay of $l$ sample periods is modelled by the subcarrier vector, $\underline{\mathbf{f}}_{k}[l]$, defined as

$$
\underline{\mathbf{f}}_{k}[l] \triangleq\left[\begin{array}{c}
\exp \left(j 2 \pi F_{k}(-l) T_{s}\right) \\
\exp \left(j 2 \pi F_{k}(1-l) T_{s}\right) \\
\vdots \\
\exp \left(j 2 \pi F_{k}\left(N_{s c}-1-l\right) T_{s}\right) \\
\underline{0}_{N_{s c}}
\end{array}\right] \in \mathcal{C}^{2 N_{s c} \times 1}
$$


where $\underline{0}_{N_{s c}}$ is a vector of $N_{s c}$ zeros [6]. The shift operator matrix, $\mathbb{J}$, is given by

$$
\mathbb{J}=\left[\begin{array}{ll}
\underline{0}_{2 N_{s c}-1}^{T} & 0 \\
\mathbb{I}_{2 N_{s c}-1} & \underline{0}_{2 N_{s c}-1}
\end{array}\right] \in \mathcal{C}^{2 N_{s c} \times 2 N_{s c}}
$$

where $\mathbb{I}_{N}$ is and $N \times N$ identity matrix and $\mathbb{J}^{l}$ neatly implements a right (down) shift by $l$ elements, accounting for the delay of the associated path.

The discretized received signal vector for the $n$-th MC-CDMA symbol interval, $\underline{x}[n] \in$ $\mathcal{C}^{2 N N_{s c} \times 1}$, is then obtained by sampling the output of the TDLs at a rate of $1 / T_{c s}$. An expression for $\underline{x}[n]$ will be derived in the next section using the diffused and point-source channel models. The vector $\underline{x}[n]$ is then fed into a suitable linear MC-CDMA array receiver (weight vector $\underline{w}_{i}$ ) to yield the decision variable $d_{i}[n]$. The decision variable is passed through a decision device to obtain the received symbol sequence $\left\{\hat{a}_{i}[n]\right\}$.

\section{Diffused SiVO Channel Modelling}

The baseband model of the diffused wireless channel is presented in Fig. 3, where the $i$-th user's signal arrives at the receiver via $K_{i}$ diffused multipath clusters. Note that each multipath becomes a cluster of rays and each cluster is made up of $W_{i j}$ space-time inseparable point-like rays [7], the $k$-th ray of $j$-th cluster of the $i$-th user having the path coefficient $\beta_{i j k}$, directionof-arrival (DOA) $\theta_{i j k}$ and timing-of-arrival (TOA) $\tau_{i j k}$. Note that $\underline{S}\left(\theta_{i j k}\right)$ is the array manifold vector for the $i$-th user's $j$-th cluster's $k$-th ray arriving at an angle of $\theta_{i j k}$, and is a function of the receiver array geometry. The TOA of the ray $\tau_{i j k}$ has a perturbation element $\tilde{\tau}_{i j k}$ about nominal TOA $\tau_{i j}$ so that $\tau_{i j k}=\tau_{i j}+\tilde{\tau}_{i j k}$. Similarly, the DOA of the ray $\theta_{i j k}$ has a perturbation element $\tilde{\theta}_{i j k}$ about nominal DOA $\theta_{i j}$ so that $\theta_{i j k}=\theta_{i j}+\tilde{\theta}_{i j k}$.

The spatio-temporal diffusion $\left(\Delta \theta_{i j}, \Delta \tau_{i j}\right), \forall j \in\left\{1, \ldots, K_{i}\right\}$ is due to the spatio-temporally unresolvable point-like rays that arrive at the receiver after diffuse reflection through perturbations $\tilde{\theta}_{i j k}$ 's and $\tilde{\tau}_{i j k}$ 's with respect to the nominal DOAs and TOAs respectively. The spatial and temporal spread of a cluster are defined in terms of the spatial resolution $\left(\theta_{\text {res }}\right)$ and temporal resolution $\left(\tau_{r e s}\right)$ of the receiver system respectively. The maximum spatial spread $\Delta \theta$ of a cluster corresponds to the angular separation of two rays which are farthest apart spatially but where the delay difference between them is less than $\tau_{\text {res }}$. Similarly, the maximum temporal spread $\Delta \tau$ corresponds to the maximum delay difference between two rays within the cluster whose spatial separation is still below $\theta_{\text {res }}[4]$. 
The baseband signal-vector at the output of the antenna array receiver, through a diffused channel, can thus be expressed as

$$
\underline{x}(t)=\sum_{i=1}^{M} \sum_{j=1}^{K_{i}} \sum_{k=1}^{W_{i j}} \beta_{i j k} \underline{S}\left(\theta_{i j k}\right) m_{i}\left(t-\tau_{i j k}\right)+\underline{\mathrm{n}}(t) \in \mathcal{C}^{N \times 1}
$$

where $\beta_{i j k}$ has absorbed the factor $\sqrt{P_{i}} \exp \left(-j 2 \pi F_{c} \tau_{i j k}\right)$.

After passing through the TDLs and discretizer, the received signal during the $n$-th symbol interval is given by

$$
\underline{x}[n]=\sum_{i=1}^{M} \sum_{j=1}^{K_{i}} \sum_{k=1}^{W_{i j}}\left(\begin{array}{c}
\beta_{i j k}\left(\underline{S}\left(\theta_{i j k}\right) \otimes \mathbb{J}^{l_{i j k}} \underline{\mathfrak{c}}\left(l_{i j k}\right)\right) \mathrm{a}_{i}[n] \\
+\beta_{i j k}\left(\underline{S}\left(\theta_{i j k}\right) \otimes\left(\mathbb{J}^{T}\right)^{N_{s c}} \mathbb{J}^{l_{i j k}} \underline{\mathfrak{c}}\left(l_{i j k}\right)\right) \mathrm{a}_{i}[n-1]+ \\
\beta_{i j k}\left(\underline{S}\left(\theta_{i j k}\right) \otimes \mathbb{J}^{N_{s c}} \mathbb{J}^{l_{i j k}} \underline{\mathfrak{c}}\left(l_{i j k}\right)\right) \mathrm{a}_{i}[n+1]
\end{array}\right)+\underline{\mathrm{n}}[n]
$$

where $\otimes$ is the Kronecker product, and $\underline{\mathfrak{c}}\left(l_{i j k}\right)$ is defined as

$$
\underline{\mathfrak{c}}\left(l_{i j k}\right) \triangleq\left[\underline{\mathbf{f}}_{1}\left[l_{i j k}\right], \ldots, \underline{\mathbf{f}}_{N_{s c}}\left[l_{i j k}\right]\right] \underline{\alpha}_{i} \in \mathcal{C}^{2 N_{s c} \times 1}
$$

The use of a first order Taylor series approximation of the array manifold vectors about the nominal DOAs simplifies the first term of Eqn. 10 to yield

$$
\begin{aligned}
& \sum_{i=1}^{M} \sum_{j=1}^{K_{i}} \sum_{k=1}^{W_{i j}}\left(\begin{array}{c}
\beta_{i j k} \underline{S}\left(\theta_{i j}\right) \otimes \mathbb{J}^{l_{i j k}} \underline{\mathfrak{c}}\left(l_{i j k}\right)+ \\
\beta_{i j k} \tilde{\theta}_{i j k} \underline{\dot{S}}\left(\theta_{i j}\right) \otimes \mathbb{J}^{l_{i j k}} \underline{\mathfrak{c}}\left(l_{i j k}\right)
\end{array}\right) \mathrm{a}_{i}[n] \\
= & \sum_{i=1}^{M} \sum_{j=1}^{K_{i}}\left[\underline{S}\left(\theta_{i j}\right) \otimes \mathfrak{C}_{i j} \mathbb{T}_{i j}, \underline{\dot{S}}\left(\theta_{i j}\right) \otimes \mathfrak{C}_{i j} \mathbb{T}_{i j}\right] \underline{g}_{i j} \mathrm{a}_{i}[n]
\end{aligned}
$$

Note that $\underline{\dot{S}}(\theta)$ is the first derivative of $\underline{S}(\theta)$ with respect to $\theta$. Code matrix $\mathfrak{C}_{i j}$, temporal matrix $\mathbb{T}_{i j}$ and cluster channel response vector $\underline{g}_{i j}$ are defined as follows

$$
\begin{aligned}
\mathfrak{C}_{i j} \triangleq\left[\underline{\mathfrak{c}}\left(l_{i j}\right), \mathbb{d} \underline{\mathfrak{c}}\left(l_{i j}\right), \ldots, \mathbb{J}^{N_{s c}-1} \mathfrak{c}\left(l_{i j}\right)\right] \in \mathcal{C}^{2 N_{s c} \times N_{s c}} \\
\mathbb{T}_{i j} \triangleq\left[\begin{array}{c}
\mathbb{O}_{\left(l_{i j}-1\right) \times W_{i j}} \\
\mathbb{I}_{W_{i j}} \\
\mathbb{O}_{\left(N_{s c}-W_{i j}-l_{i j}+1\right) \times W_{i j}}
\end{array}\right] \in \mathcal{C}^{N_{s c} \times W_{i j}} \\
\underline{g}_{i j} \triangleq\left[\underline{\beta}_{i j}^{T},\left(\underline{\beta}_{i j} \odot \underline{\tilde{\theta}}_{i j}\right)^{T}\right]^{T} \in \mathcal{C}^{2 W_{i j} \times 1}
\end{aligned}
$$

where $\mathbb{O}_{N \times M}$ is an $N \times M$ matrix consisting of all zeros, $\odot$ is the Hadamard product, $\underline{\beta}_{i j}=$ $\left[\beta_{i j 1}, \beta_{i j 2}, \ldots, \beta_{i j W_{i j}}\right]$ and $\underline{\tilde{\theta}}_{i j}=\left[\tilde{\theta}_{i j 1}, \tilde{\theta}_{i j 2}, \ldots, \tilde{\theta}_{i j W_{i j}}\right]$. 
By defining the diffused STAR (ㅁatial-temporal array) manifold vector as

$$
\underline{\mathfrak{h}}_{i j} \triangleq\left[\underline{S}\left(\theta_{i j}\right) \otimes \mathfrak{C}_{i j} \mathbb{T}_{i j}, \underline{\dot{S}}\left(\theta_{i j}\right) \otimes \mathfrak{C}_{i j} \mathbb{T}_{i j}\right] \underline{g}_{i j} \in \mathcal{C}^{2 N_{s c} \times 1}
$$

the received signal can be written as

$$
\begin{aligned}
\underline{x}[n] & =\sum_{i=1}^{M} \sum_{j=1}^{K_{i}}\left[\underline{\mathfrak{h}}_{i j, p r e v}, \underline{\mathfrak{h}}_{i j}, \underline{\mathfrak{h}}_{i j, n e x t}\right]\left[\begin{array}{c}
\mathrm{a}_{i}[n-1] \\
\mathrm{a}_{i}[n] \\
\mathrm{a}_{i}[n+1]
\end{array}\right]+\underline{\mathrm{n}}[n] \\
& =\sum_{i=1}^{M} \mathbb{H}_{i} \underline{\mathrm{a}}_{i}[n]+\underline{\mathrm{n}}[n] \\
& =\mathbb{H} \underline{\mathbf{a}}[n]+\underline{\mathrm{n}}[n]
\end{aligned}
$$

where

$$
\begin{aligned}
& \underline{\mathfrak{h}}_{i j, \text { prev }}=\left(\mathbb{I}_{N_{s c}} \otimes\left(\mathbb{J}^{T}\right)^{N_{s c}}\right) \underline{\mathfrak{h}}_{i j} \\
& \underline{\mathfrak{h}}_{i j, n e x t}=\left(\mathbb{I}_{N_{s c}} \otimes \mathbb{J}^{N_{s c}}\right) \underline{\mathfrak{h}}_{i j} \\
& \underline{\mathbf{a}}_{i}[n]=\left[\mathbf{a}_{i}[n-1], \mathbf{a}_{i}[n], \mathbf{a}_{i}[n+1]\right]^{T} \\
& \underline{\mathbf{a}}[n]=\left[\underline{\mathbf{a}}_{1}^{T}[n], \underline{\mathbf{a}}_{2}^{T}[n], \ldots, \underline{\mathbf{a}}_{M}^{T}[n]\right]^{T}
\end{aligned}
$$

Note that in Eqn. 17, the matrix $\mathbb{H}$ is the composite channel matrix defined as

$$
\mathbb{H} \triangleq\left[\mathbb{H}_{1}, \mathbb{H}_{2}, \ldots, \mathbb{H}_{M}\right]
$$

with $\mathbb{H}_{i} \triangleq\left[\underline{h}_{i, \text { prev }}, \underline{h}_{i}, \underline{h}_{i, \text { next }}\right]$, where the composite channel vector $\underline{h}_{i}$ is a linear combination of $\underline{\mathfrak{h}}_{i j}$ 's given by

$$
\underline{h}_{i}=\sum_{j=1}^{K_{i}} \underline{\mathfrak{h}}_{i j}
$$

and $\underline{h}_{i, p r e v}, \underline{h}_{i, \text { next }}$ are defined similarly.

It is important to point out that for the point-source assumption, Eqn. 10 reduces to the following expression

$$
\underline{x}[n]=\sum_{i=1}^{M} \sum_{j=1}^{K_{i}}\left(\begin{array}{c}
\mathbb{H}_{i j, p r e v} \beta_{i j} \underline{\alpha}_{i} \mathrm{a}_{i}[n-1] \\
+\mathbb{H}_{i j} \beta_{i j} \underline{\alpha}_{i} \mathrm{a}_{i}[n] \\
+\mathbb{H}_{i j, n e x t} \beta_{i j} \underline{\alpha}_{i} \mathrm{a}_{i}[n+1]
\end{array}\right)+\underline{\mathrm{n}}[n]
$$


where

$$
\begin{aligned}
& \mathbb{H}_{i j}=\underline{S}\left(\theta_{i j}\right) \otimes\left(\mathbb{J}^{l_{i j}}\left[\underline{\mathbf{f}}_{1}\left[l_{i j}\right], \ldots, \underline{\mathbf{f}}_{N_{s c}}\left[l_{i j}\right]\right]\right) \\
& \mathbb{H}_{i j, p r e v}=\underline{S}\left(\theta_{i j}\right) \otimes\left(\left(\mathbb{J}^{T}\right)^{N_{s c}} \mathbb{J}^{l_{i j}}\left[\underline{\mathbf{f}}_{1}\left[l_{i j}\right], \ldots, \underline{\mathbf{f}}_{N_{s c}}\left[l_{i j}\right]\right]\right) \\
& \mathbb{H}_{i j, n e x t}=\underline{S}\left(\theta_{i j}\right) \otimes\left((\mathbb{J})^{N_{s c}} \mathbb{J}^{l_{i j}}\left[\underline{\mathbf{f}}_{1}\left[l_{i j}\right], \ldots, \underline{\mathbf{f}}_{N_{s c}}\left[l_{i j}\right]\right]\right)
\end{aligned}
$$

The composite channel vector for the point-source model is thus given by

$$
\underline{h}_{i}=\sum_{j=1}^{K_{i}} \mathbb{H}_{i j} \beta_{i j} \underline{\alpha}_{i}
$$

and the point-source composite channel matrix can similarly be formulated using Eqn. 18 .

From the above, it can be seen that the diffused SIVO channel model proposed in this section is a generic approach and point-source channel model is just a special case, obtained by applying the point-source assumption.

\section{Simulation Studies}

Computer simulations are presented in this section to observe the effect of spatio-temporal diffusion on arrayed MC-CDMA receivers and to highlight the key benefits of using the diffused vector channel modelling in arrayed MC-CDMA systems. The two receivers investigated in this study are the RAKE receiver and the Decorrelating receiver. RAKE receiver performance will act as a lower bound whereas that of the space-time Decorrelating receiver will be the upper limit for arrayed MC-CDMA receivers' performance in these simulations. Weight vectors for both these receivers (assuming user 1 to be the desired user) expressed as a function of the estimated STAR manifold vectors are

\begin{tabular}{|c|c|}
\hline RAKE Receiver & $\underline{w}_{1}=\underline{\hat{h}}_{1}$ \\
\hline Decorrelating Receiver & $\underline{w}_{1}=\operatorname{col}_{2}\left(\left(\widehat{\mathbb{H}}^{\dagger}\right)^{T}\right)$ \\
\hline
\end{tabular}

where $\operatorname{col}_{i}(\mathbb{A})$ selects the $i$-th column of $\mathbb{A}$ and $(\cdot)^{\dagger}$ gives the pseudo-inverse. Note that $\underline{\underline{h}}_{1}$ is the estimated composite channel vector obtained using Eqn. 19 or Eqn. 21, and $\widehat{\mathbb{H}}$ is the estimated composite channel matrix obtained from Eqn. 18.

The simulation parameters of the MC-CDMA system under consideration are given in Table I. The number of co-channel users $M=3$ and the interfering users have the same power as the desired user (near-far ratio $=0 \mathrm{~dB}$ ). A frame of 1000 symbols is collected each time 
for processing (i.e., $L=1000$ ) and the arrayed receiver employs a 5-element uniform linear array (ULA) with half-wavelength inter-element spacing. The channel is assumed to be diffused spatially and temporally, and the nominal DOAs and nominal TOAs of the multipath clusters are assumed to be pre-estimated (e.g. using pilot-based approaches [8], or the method proposed in [9]).

\section{A. BER Performance of Arrayed MC-CDMA Receivers}

Bit error rate (BER) at the receiver output is a useful metric for the comparison of arrayed MC-CDMA receivers. In the first simulation study, the point-source and the proposed diffused SIVO channel models are used to model a spatio-temporally diffused channel and the BER is computed at the output of arrayed MC-CDMA receivers. The channel is assumed to be diffused $10^{\circ}$ spatially and $7 T_{c}$ temporally in each of the five multipath clusters.

Fig. 4 depicts the BER observed at the output of the RAKE receiver as the input Signalto-Noise Ratio (SNR) varies from $0 \mathrm{~dB}$ to $20 \mathrm{~dB}$. Two scenarios are compared: in the first case the receiver ignores spatial and temporal diffusion by using the point-source vector channel model, whereas in the second case the diffused channel model is used. Point-source channel model does not take into account the spatial and temporal diffusion of the multipath clusters, and hence fails to model the wireless channel accurately. On the other hand, the diffused SIVO channel model considers the spatio-temporal diffusion of the multipath clusters and gives bettter BER performance.

BER performance of the Decorrelating receiver is shown in Fig. 5, using the point-source and the diffused SIVO channel models respectively. Once again, a performance degradation is observed when using the point-source channel model. On the contrary, using a Decorrelating receiver based on the diffused channel model gives better BER, especially at higher SNRs. It is evident from both Fig. 4 and Fig. 5 that considerable diffused channel interference is cancelled by employing the proposed diffused channel model in the design of arrayed MC-CDMA receiver weights. 


\section{B. SNIR ${ }_{\text {out }}$ Performance of Arrayed MC-CDMA Receivers}

Another metric to compare the performance of arrayed MC-CDMA receivers is the $\mathrm{SNIR}_{\text {out }}$

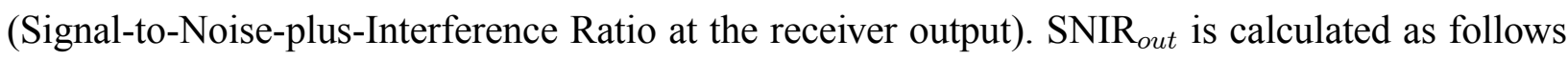

$$
\operatorname{SNIR}_{\text {out }}=\frac{\underline{w}_{1}^{H} \mathbb{R}_{11} \underline{w}_{1}}{\underline{w}_{1}^{H} \mathbb{R}_{N+I} \underline{w}_{1}}
$$

where $\mathbb{R}_{11}$ is the covariance matrix of the desired user (assumed to be user 1 , without any loss of generality) and $\mathbb{R}_{N+I}$ is the covariance matrix of noise plus interference [10]. Therefore, the overall data covariance matrix is given by

$$
\mathbb{R}_{x x}=\mathbb{R}_{11}+\mathbb{R}_{N+I}
$$

The performance of both RAKE and Decorrelating receivers will now be studied with respect to their respective $\mathrm{SNIR}_{\text {out }}$. Once again, point-source reception that ignores the presence of spatial-temporal diffusion is compared to reception using the diffused channel model for both receivers (RAKE and Decorrelating). Assume the wireless channel to be diffused with $5^{\circ}$ spatial spread and temporal spread equivalent to $3 T_{c}$ in each of the five multipath clusters.

Fig. 6 depicts the SNIR out at the output of the arrayed MC-CDMA receivers as a function of the input Signal-to-Noise Ratio (SNR). The SNIR $_{\text {out }}$ of the Decorrelating receiver based on the proposed diffused channel model increases linearly with input SNR. Although the channel is severely diffused spatio-temporally, the proposed approach models the diffused channel accurately to cancel the diffused channel interference and yield good performance. However, the Decorrelating receiver based on point-source channel model exhibits saturation at a relatively low input SNR of around $5 \mathrm{~dB}$, due to uncancelled diffused channel interference. The RAKE receivers based on the diffused and point-source channel models show characteristic low performance and saturation, but the reception based on diffused channel model is slightly better

Similar SNIR $_{\text {out }}$ vs. input SNR plots are considered for a channel with $10^{\circ}$ spatial and $6 T_{c}$ temporal diffusion in Fig. 7. Since each multipath cluster in this channel has increased spatial and temporal spread, the performance of the receivers degrades slightly in comparison with Fig. 6. To explore this in more detail, the next sub-section will focus on the impact of varying degrees of spatial and spatio-temporal diffusion on the performance of arrayed MC-CDMA receivers. 


\section{Impact of Spatio-Temporal Diffusion on Receiver Performance}

To analyse the effect of spatio-temporal diffusion on common receivers, consider the $\mathrm{SNIR}_{\text {out }}$ of arrayed MC-CDMA receivers as the spatial and/or temporal spread varies. Initially the effect of spatial spread only (zero temporal spread) on the SNIR $_{\text {out }}$ performance is presented. Fig. 8 depicts the RAKE receiver SNIR $_{\text {out }}$ performance using the diffused and point-source channel models, whereas Fig. 9 gives the corresponding plots for the Decorrelating receiver. Both plots were produced at an input SNR of $10 \mathrm{~dB}$.

The more severe space-time diffused channel, which is diffused spatially as well as temporally, is considered next. Fig. 10 depicts the SNIR $_{\text {out }}$ for the RAKE receiver versus varying degrees of spatio-temporal spread assuming an input SNR of $10 \mathrm{~dB}$. The point-source channel model-based receiver's performance degrades very badly, while that based on the diffused channel model is relatively unaffected by spatio-temporal diffusion. Similar plots for the performance of the Decorrelating receiver are presented in Fig. 11.

It can be observed the point-source channel model-based receiver's $\mathrm{SNIR}_{\text {out }}$ depreciates rapidly but the receiver based on the diffused channel model shows very little performance degradation. When comparing the Decorrelating and RAKE receiver performances in terms of SNIR out, it can be seen that the Decorrelating receiver is about four times better than the RAKE receiver. However, Decorrelating receiver achieves this at the cost of additional computational complexity [11]. For the Decorrelating receiver, it is very important to use the diffused channel model for weight formulation, because its $\mathrm{SNIR}_{\text {out }}$ performance degrades very rapidly with increasing spatio-temporal spread as compared to the RAKE receiver.

\section{CONCLUSiOnS}

In this paper, the space-time diffused wireless channel experienced by arrayed MC-CDMA systems is modelled. Localized scattering of the multipaths motivates the frequency selective wireless channel to be modelled as a diffused vector channel. The space-time diffused vector channel for an arrayed MC-CDMA system is characterized in terms of the spatial and temporal parameters. Simulation results show that the use of this diffused channel modelling in arrayed MC-CDMA receivers yields better bit error rate (BER) and $\mathrm{SNIR}_{\text {out }}$ performance than receivers that ignore the presence of spatial and temporal diffusion. 


\section{REFERENCES}

[1] G.J.Foschini and M.J.Gans, "On Limits of Wireless Communications in a Fading Environment when Using Multiple Antennas," IEEE Wireless Personal Communications, vol. 6, no. 3, pp. 311-335, 1998.

[2] R. Esmailzadeh, M. Nakagawa, and A. Jones, “TDD-CDMA for the 4th generation of wireless communications," Wireless Personal Communications, vol. 10, no. 4, pp. 8-15, 2003.

[3] D. J. Sadler and A. Manikas, "MMSE multiuser detection for array multicarrier DS-CDMA in fading channels," IEEE Transactions on Signal Processing, vol. 53, no. 7, pp. 2348-2358, 2005.

[4] T. S. Naveendra and A. Manikas, "Subspace receiver techniques for DS-CDMA systems in space diffused vector channels," WSEAS Transactions on Communications, vol. 3, no. 2, pp. 590-594, 2004.

[5] T. S. Naveendra and A. Manikas, "Blind estimation of diffused multipath vector channels for DS-CDMA systems," 1st International Symposium on Wireless Communication Systems 2004, pp. 41-45, 2004.

[6] H. H. Peh and A. Manikas, "An investigative study of cyclic prefix-free MC-CDMA Arrayed-MIMO Communication Systems," International Symposium on Wireless Pervasive Computing 2007, 2007.

[7] K. I. Pedersen, P. E. Mogensen, and B. H. Fleury, "A stochastic model of the temporal and azimuthal dispersion seen at the base station in outdoor propagation environments," IEEE Transactions on Vehicular Technology, vol. 49, no. 2, pp. 437-447, 2000.

[8] B. Muquet, Z. Wang, G. B. Giannakis, M. de Courville, and P. Duhamel, "Cyclic Prefixing or Zero Padding for Wireless Multicarrier Transmissions?," IEEE Transactions on Communications, vol. 50, no. 12, pp. 2136-2148, 2002.

[9] T. Zhang and A. Manikas, "OFDM-CDMA Array System for Interference Cancellation," Proceedings of the 7th Hellenic European Conference on Computer Mathematics and its Applications, 2005.

[10] D. J. Sadler and A. Manikas, "Blind Reception of Multicarrier DS-CDMA using Antenna Arrays ," IEEE Transactions on Wireless Communications, vol. 2, no. 6, pp. 1231-1239, 2003.

[11] S. Verdu, Multiuser Detection. Cambridge UK: Cambridge University Press, 1998. 


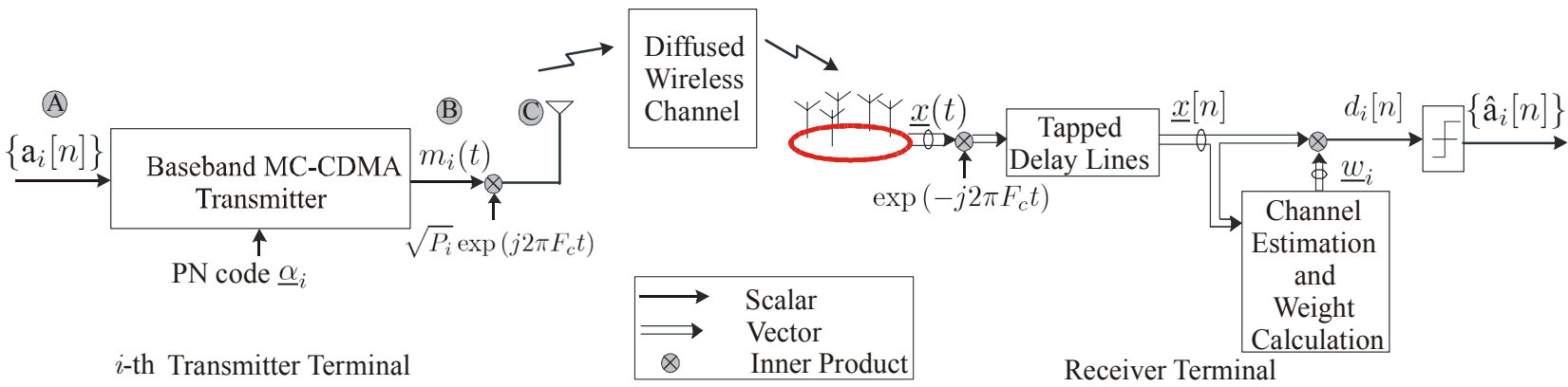

Fig. 1. Arrayed MC-CDMA communication system operating in a space-time diffused wireless channel

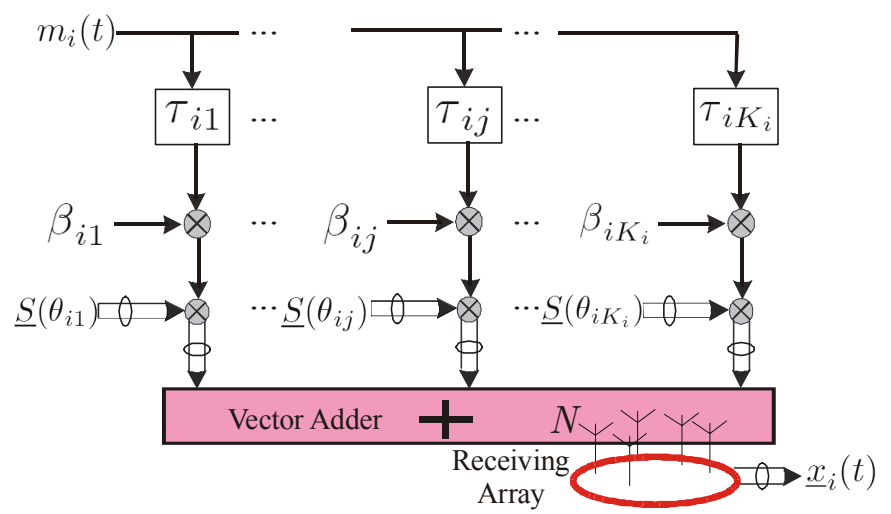

Fig. 2. Point-source Scalar-Input-Vector-Output (SIVO) baseband channel model for the $i$-th user as a function of the array manifold vectors

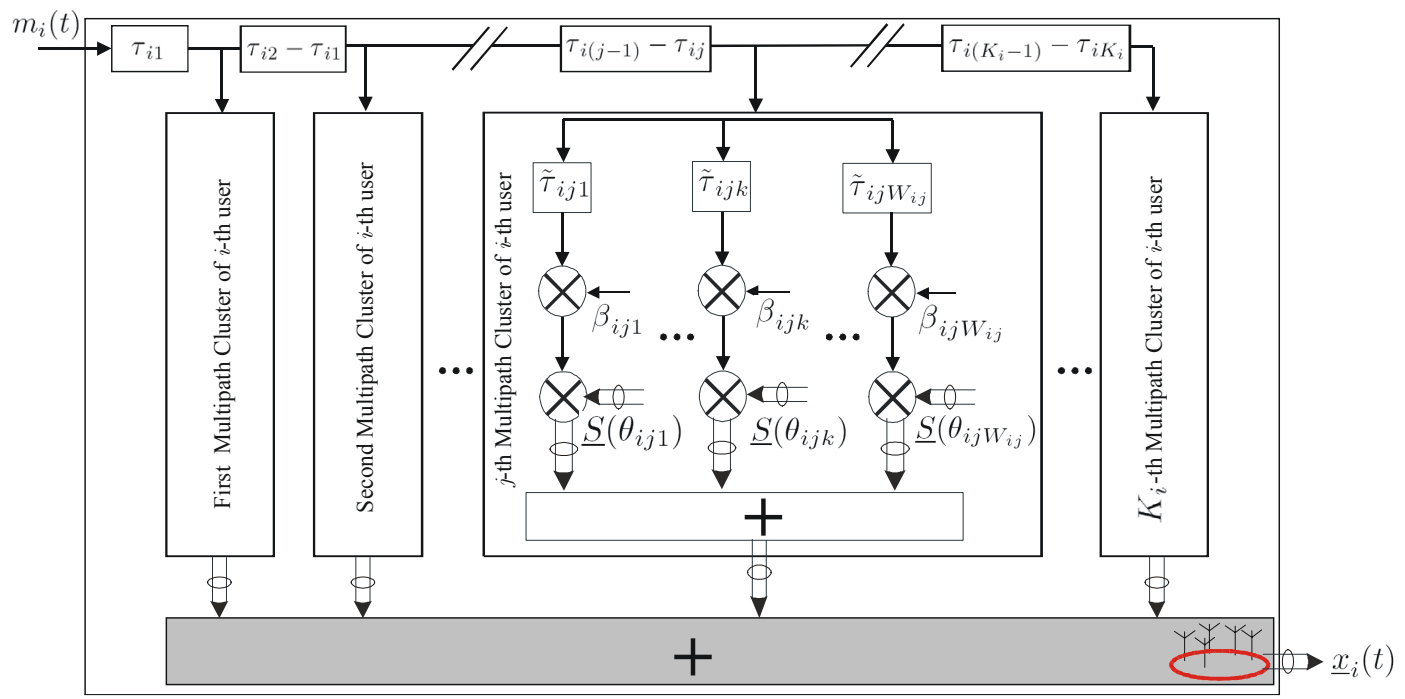

Fig. 3. Diffused Scalar-Input-Vector-Output (SIVO) baseband channel model for the $i$-th user 


\begin{tabular}{|c|c|}
\hline Parameters & Specification \\
\hline Number of Subcarriers $N_{s c}$ & 64 \\
\hline Signal Constellation & BPSK \\
\hline Inter-subcarrier Spacing $(\Delta F)$ & $781.25 \mathrm{kHz}$ \\
\hline System Bandwidth & $50 \mathrm{MHz}$ \\
\hline Carrier Frequency $\left(F_{c}\right)$ & $5 \mathrm{GHz}$ \\
\hline
\end{tabular}

TABLE I

SimULATION PARAMETERS

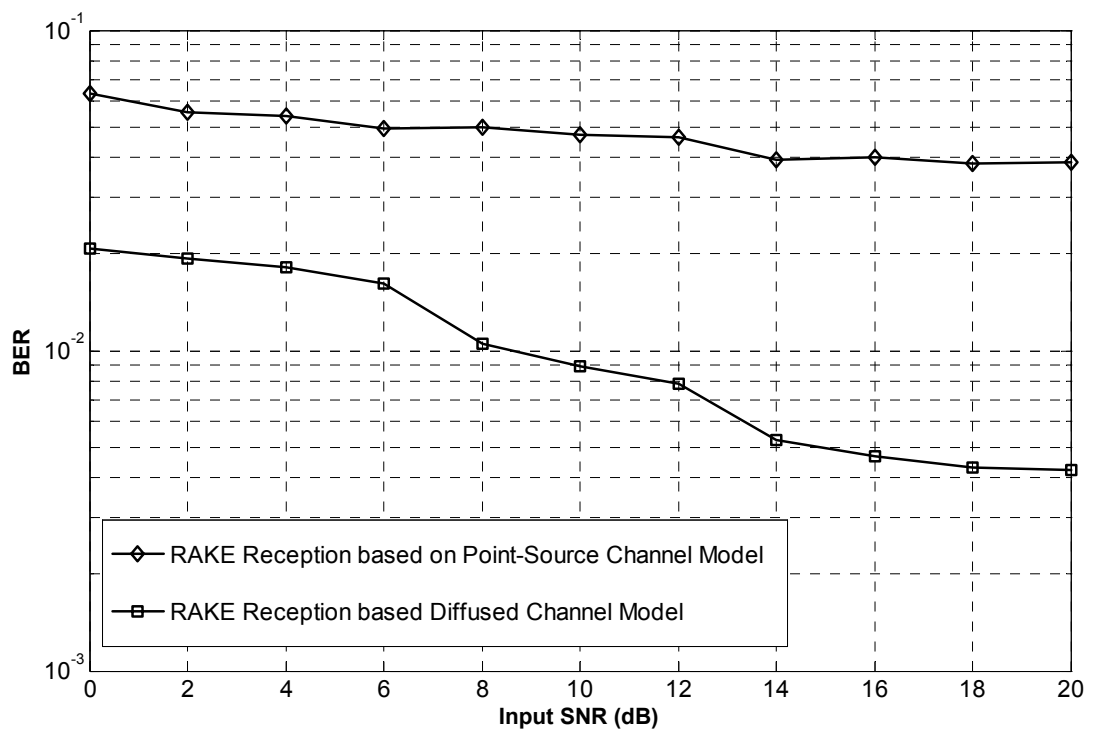

Fig. 4. RAKE receiver: BER versus Input SNR plots using the diffused and point-source channel models in a $\left(10^{\circ}, 7 T_{c}\right)$ diffused channel 


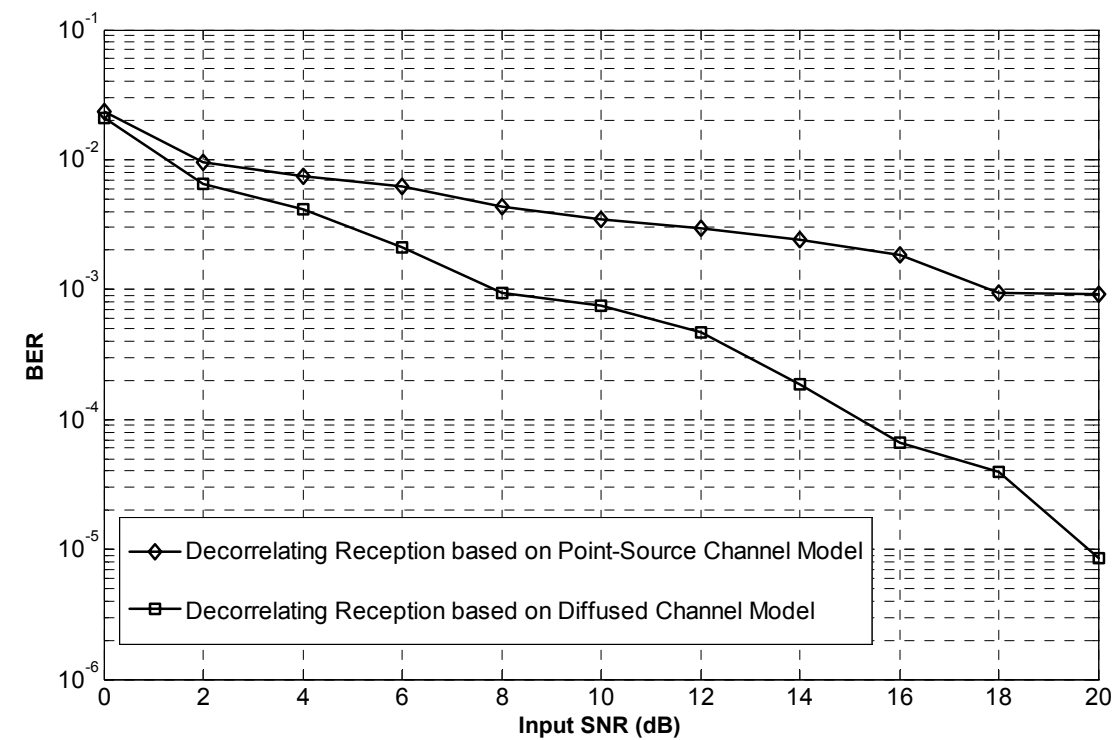

Fig. 5. Decorrelating receiver: BER versus Input SNR plots using the diffused and point-source channel models in a $\left(10^{\circ}, 7 T_{c}\right)$ diffused channel

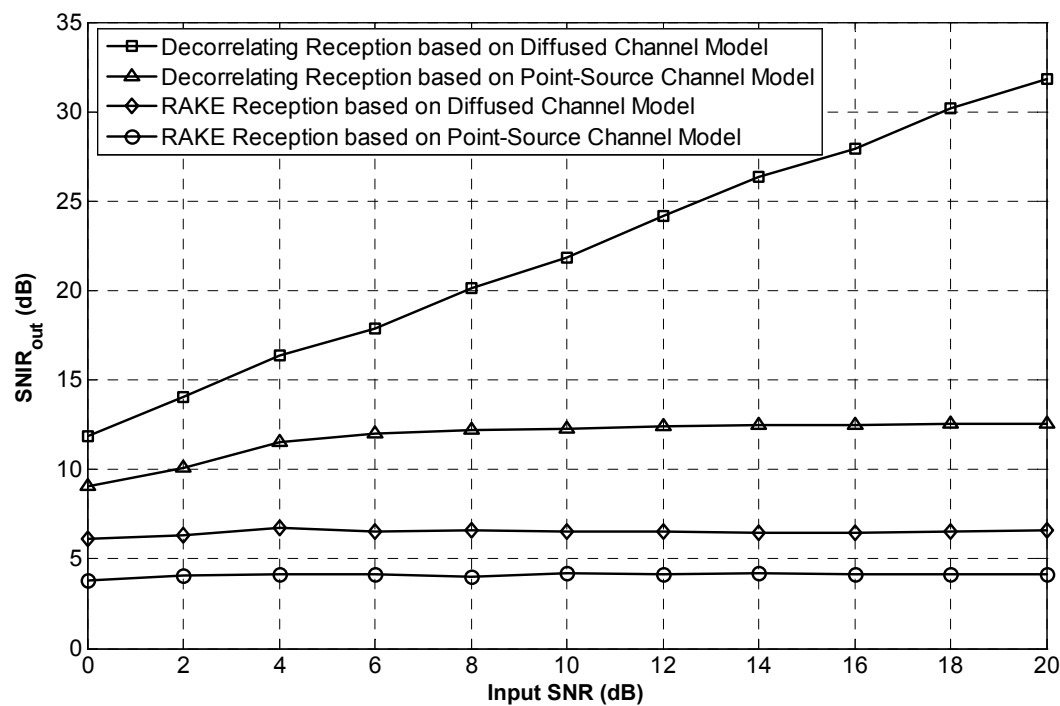

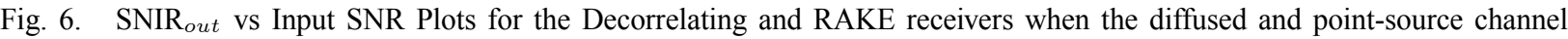
models are used in a $\left(5^{\circ}, 3 T_{c}\right)$ diffused channel 


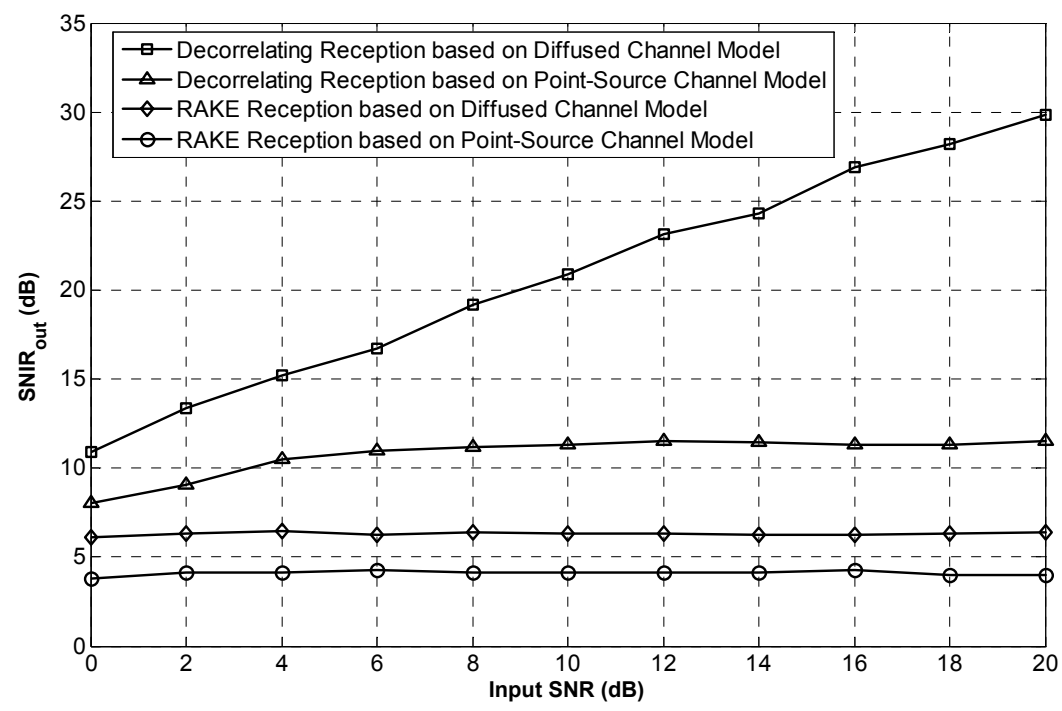

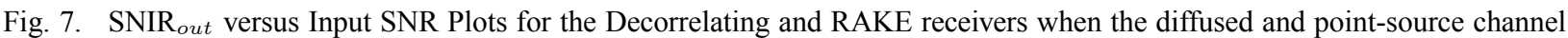
models are used in a $\left(10^{\circ}, 6 T_{c}\right)$ diffused channel

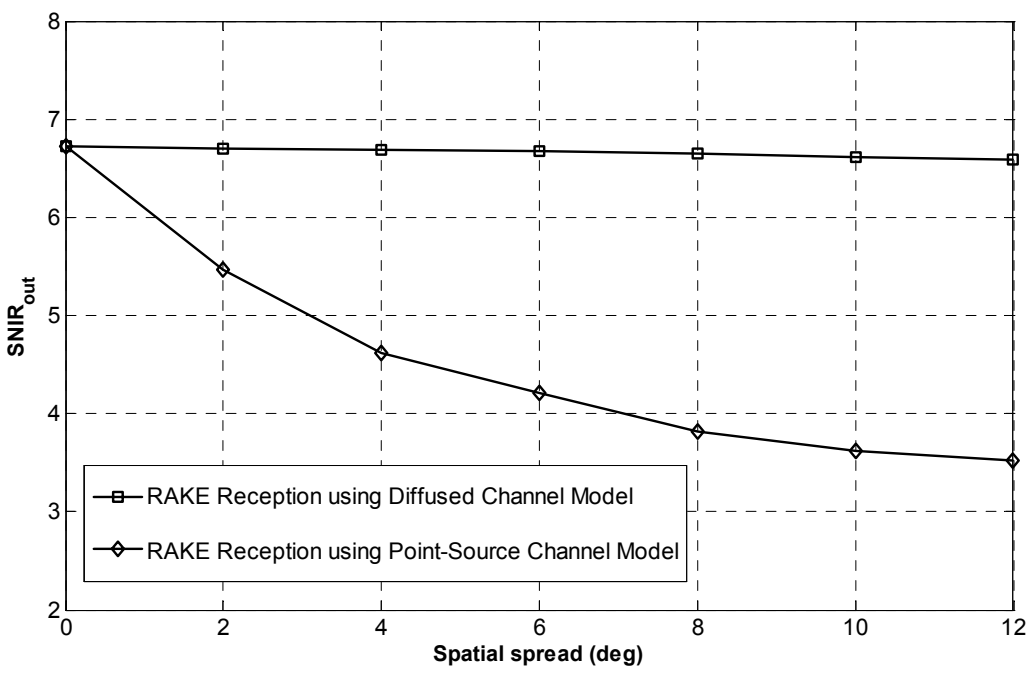

Fig. 8. RAKE receiver: $\mathrm{SNIR}_{\text {out }}$ performance against multipath spatial (only) spread (Input $\mathrm{SNR}=10 \mathrm{~dB}$ ) 


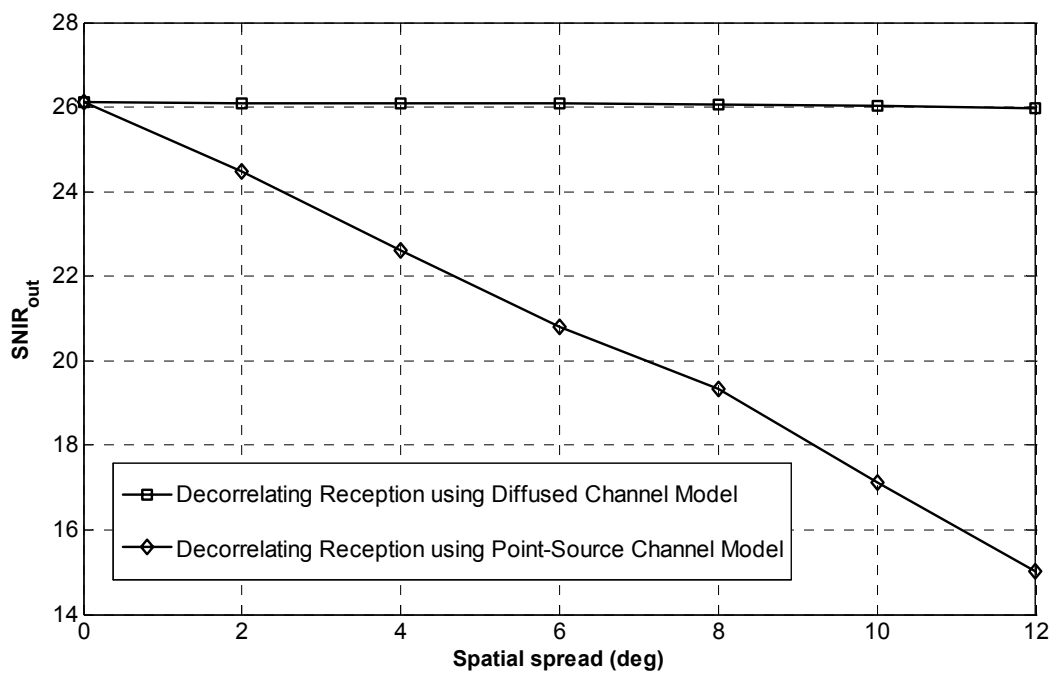

Fig. 9. Decorrelating receiver: $\mathrm{SNIR}_{\text {out }}$ performance against multipath spatial (only) spread (Input $\mathrm{SNR}=10 \mathrm{~dB}$ )

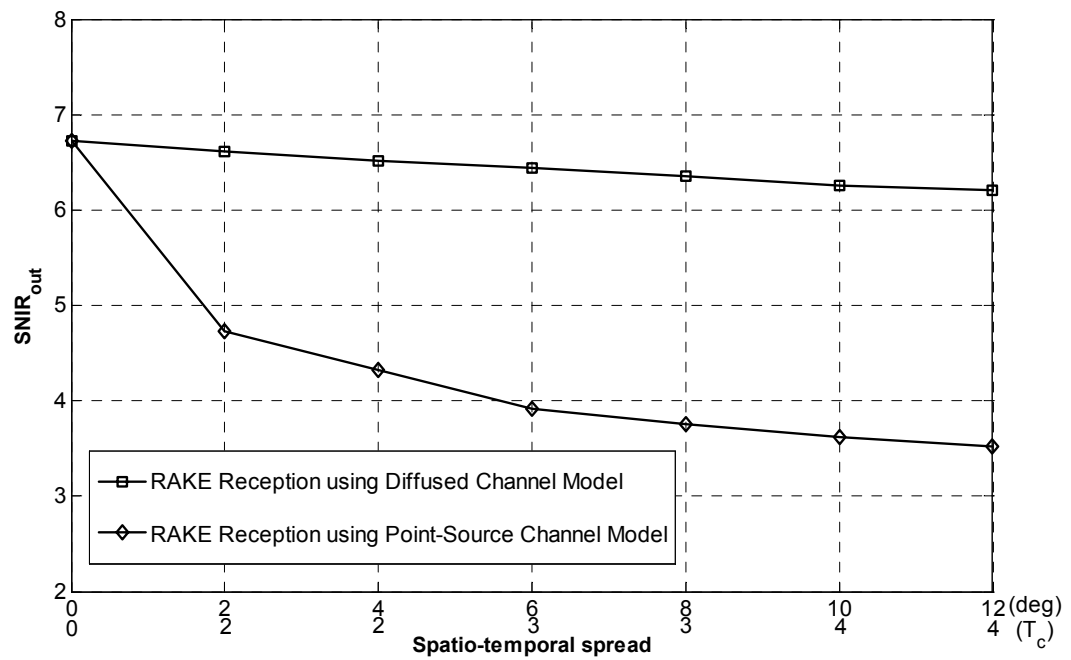

Fig. 10. RAKE receiver: $\mathrm{SNIR}_{\text {out }}$ performance against multipath spatio-temporal spread (Input $\mathrm{SNR}=10 \mathrm{~dB}$ ) 


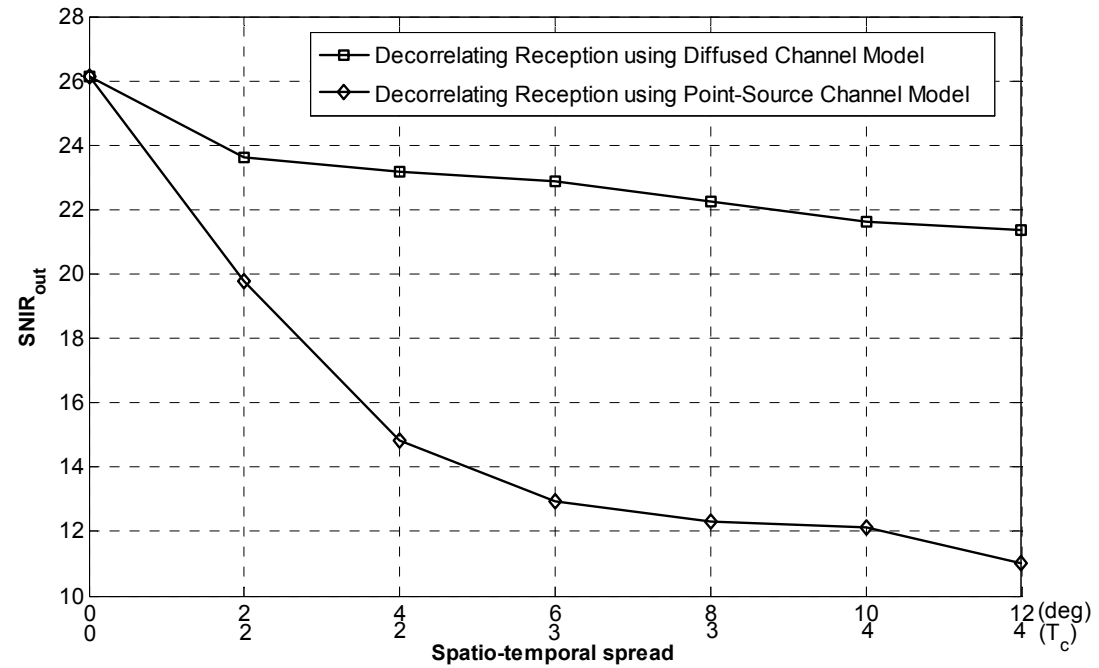

Fig. 11. Decorrelating receiver: $\mathrm{SNIR}_{\text {out }}$ performance against multipath spatio-temporal spread (Input $\mathrm{SNR}=10 \mathrm{~dB}$ ) 